\title{
The efficacy of St. John's wort (SJW, hypericum perforatum) oil macerates on intractable skin lesions of patients with idiopathic granulomatous mastitis (IGM): Preliminary results
}

\author{
Sema Yuksekdag ${ }^{1}$ \\ ${ }^{1}$ Umraniye Training and Research Hospital
}

June 2, 2020

\begin{abstract}
Purpose: St. John's wort (SJW, hypericum perforatum) has a long history of medicinal use mainly for its antidepressive effect and for wound healing. However, to the best of our knowledge, this is the first clinical study evaluating the effects of topical SJW oil macerates on the intractable skin lesions of idiopathic granulomatous mastitis (IGM). Materials and Methods: SJW oil massage (two times daily for two minutes) was recommended between 2016 and 2019, only for patients with persistent or intractable skin lesions, after complete regression of granulomatous mass with two cycles of high-dose oral steroid and empiric antibiotic. Skin lesions were assessed and graded before and after treatment, as clear, mild, moderate and severe. A miniquestionnaire was also filled in for each patient at the end of six-week treatment. Results: A total of 21 patients with persistent IGM lesions used SJW oil after completion of the standard treatment protocol. All patients were women, and the mean age was 36.6 years. Hyperemia (100\%), scaling (61\%), induration $(52 \%)$ and ulcer $(28 \%)$ were the predominant skin lesions, Distribution of the lesions according to the degree of severity were as mild, moderate and severe, and seen in $35 \%, 41 \%$ and $23 \%$ of the patients, respectively. When compared with pre-treatment scores, there were very significant regression (clear: $76.4 \%$, mild: 17.6\%, moderate: $3.9 \%$, severe: $1.9 \%$; $<0.001$ for each). The overall success rate (total clearance plus mild signs) of treatment was $94 \%$. Conclusion: SJW oil massage seems to be very effective in patients with IGM-related persistent skin lesions.
\end{abstract}

\section{What is already known about this topic? What does this article add?}

Idiopathic granulomatous mastitis (IGM) is a recurrent, chronic, nonspecific inflammatory disease of the breast, affecting mostly women of child-bearing age. It usually presents with a firm solitary mass associated with inflammatory skin lesions. Simple drainages, broad spectrum antibiotics and systemic corticosteroids are the mainstay of the treatment, but even the granulomatous mass might get smaller or disappear, skin lesions can still persist after treatment. St John's wort (SJW) is a herbal remedy, extracted from the flowers and leaves of the plant Hypericum perforatum and has been used over many centuries as a traditional medicine for wound healing. However, to the best of our knowledge, this is the first clinical study evaluating the efficacy of topical SJW oil macerates on the intractable skin lesions of IGM. Preliminary results are highly promising.

Reflective questions

-How come St John's oil has all of antimicrobial, anti-inflammatory, antioxidant and anticancer activities?

-Why we call hypericum perforatum as St. John's wort? Any historical event?

-What are the effects of SJW on skin wounds?

-What are the choices of middle-aged sexually active woman with idiopathic granulomatous mastitis (IGM)related intractable skin lesions and scars? Any herbal choice? 


\section{Introduction}

St. John's wort (SJW, hypericum perforatum) has been intensively investigated and used for its antidepressive activity, but mostly overlooked dermatological applications also have a long tradition. ${ }^{1}$ Topical preparations of SJW has been recommended for skin abrasions or bruises, burns, ulcers, myalgia and intractable wounds. ${ }^{2}$ Two important constituents, naphtho-dianthrones (hypericin, flavonoids) and phloro-glucinols (hyperforin), are proven to have antimicrobial, anti-inflammatory, antioxidant and anticancer activities. ${ }^{1}$ Hyperforin has been shown to stimulate growth and differentiation of keratinocytes, and hypericin is now increasingly being used in the treatment of psoriasis and skin cancer as a photosensitizer. ${ }^{3,4}$

We had knowledge about this medication when we had positive feedback from our patients with idiopathic granulomatous mastitis (IGM) who have persistent skin lesions such as unfading hyperemia or deeper ulcers and used topical oil forms of SJW on their own, without prescription. IGM is a chronic, nonspecific inflammation of the breast, affecting mostly middle-aged parous women. ${ }^{5}$ It may present as a firm solitary mass associated with an inflammatory skin fistulae or an abscess. ${ }^{6}$ Hyperemia, erythema and cellulitis are also common. A core needle biopsy (CNB) is mandatory to exclude malignancy. Most surgeons have limited experience in managing this clinic entity and the disease has a high recurrence rate. Simple drainages, broad spectrum antibiotics and systemic corticosteroids are the mainstay of the treatment, since surgery does not prevent recurrence and cosmetic results are not satisfactory. ${ }^{7}$ Although mass in the breast might get smaller or disappear, skin lesions can still persist after treatment.

In the present study, we evaluated the effectiveness of SJW on intractable skin lesions of patients with IGM. To the best of our knowledge, after a wide-range Pub-Med search, this is the first trial on this topic.

\section{Materials and Methods}

The study was approved by our institutions's Ethics' Committe (SBU/21.11.2018/B.10.1.TKH.4.34.H.GP.0.01/137). Patients' written consents were also taken after explanation of the expected effects and possible adverse effects of SJW oil macerate. Pre- and post-treatment pictures were taken for follow-up and educational purposes, and used with permission. In patients with suspected IGM, ultrasound (US) was carried out on all, and mammography (MMG) was obtained from the ones [?] 40 years. Magnetic resonance (MR) scan was also used for the differential diagnosis in complicated cases. The diagnosis was confirmed by CNB. All abscesses [?]2 $\mathrm{cm}$ were drained. In all patients with infectious or inflammatory signs, a broad spectrum empiric antibiotic was employed for at least 10 days. Following the definitive diagnosis of IGM was obtained, all used oral corticosteroid (Prednisolone $16 \mathrm{mg}$, Mustafa Nevzat Ilac San AS, Istanbul) treatment with the same high-dose as $64 \mathrm{mg} /$ day for the first four weeks, and then the dose was tapered in the following 2 weeks. In persistent or recurrent cases, the same treatment cycle was used one more time, following a 4-week interval. All IGM patients presenting with intractable skin lesions between May 2016 and September 2019 were included.

The study was designed prospectively, but all data were interrogated retrospectively, and all patients who completed a second cycle of treatment and still have a skin lesion were started on SJW treatment. SJW oil massage (two times daily, for two minutes) was prescribed to all with on-going skin lesions such as hyperemia/erythema, scaling, induration, fissure/ulceration. Ready-made SJW olive oil macerates $(280 \mathrm{~g}$ fresh SJW flowers macerated for 28 days with $500 \mathrm{ml}$ olive oil and exposed to sunlight during extraction), containing pseudohypericin $(0.1-3.3 \mu \mathrm{g} / \mathrm{g})$, flavonoid $(0.35-3.5 \mu \mathrm{g} / \mathrm{g})$, hypericin $(0.3-6.6 \mu \mathrm{g} / \mathrm{g})$ and hyperforin $(1-2.4 \mu \mathrm{g} / \mathrm{g})$ in different trade-marks were used, and patients were free to choose one. All of these patients were recalled for control at two-week intervals and were asked for any possible side-effects. The treatment was stopped when the lesion disappears or at the end of 6 weeks. Patients without follow-up were excluded from the study.

Severity of erythema, scaling, induration and fissure/ulcer were evaluated and graded as previously cited (Table 1). ${ }^{8}$ The definitions of the lesions are as following:

- Erythema: Redness of the skin or mucous membranes, caused by hyperemia (increased blood flow) in 
superficial capillaries.

- Scaling (desquamation): Loss of the outer layer of the epidermis. The skin appears dry and cracked.

- Induration: Hardening of an area of the body as a reaction to inflammation, hyperemia or neoplastic infiltration.

- Fissure/ulcer: A linear-like cleavage or sore of skin, extending into the dermis.

At the end of the treatment, a mini-questionnaire form including 3 simple questions was filled in for each patient. The questions were:

- Did you apply the SJW oil on your skin lesion two times daily for two minutes with gentle massage, and continue the treatment for 6 weeks? Please answer as yes or no.

- Did you experience any side effects such as itching, burning or prickling, increase in hyperemia, deepining of fissures etc.? Please answer as yes or no, if yes, please specify

- Are you satisfied with the treatment? Please answer as yes or no, if no, please specify

Statistical analysis was performed using SPSS Statistics 20 software. Continuous variables were compared using t-test. Categorical variables were compared using Fisher's exact test. $\mathrm{P}<0.05$ was considered statistically significant.

\section{Results}

A total of 21 patients with persistent or recurrent IGM lesions used SJW oil after completion of the standard treatment protocol. All patients were women, and the mean age was 36.6 years (range, 21-52). Most of them $(\mathrm{n}=19,90.4 \%)$ were in the reproductive age (mean 32.4 years; range 21-42, $\mathrm{p}<0.001)$. Sixteen had a lactation history over one year $(76.1 \%, \mathrm{p}<0.001)$. Four patients were nulliparous $(19 \%)$ and all others $(80.9 \%)$ had at least one child (mean, 3 children, range 1-7, p $<0.001$ ). Nineteen percent of the patients had a history of oral contraceptive use $(n=4)$, and another three were using antidepressants $(14.2 \%)$. Two patients had controlled diabetes mellitus and four had a smoking history (9.5\% and $19 \%$, respectively). There was no endocrinologic or autoimmune diseases, except thyroid disorders in two patients $(9.5 \%)$.

Physical examination revealed persistent hyperemia/erythema $(\mathrm{n}=21,100 \%)$, scaling $(\mathrm{n}=13,61.9 \%)$, induration $(\mathrm{n}=11,52.3 \%)$ and fissure/ulcer $(\mathrm{n}=6,28.5 \%)$. Distributions of the lesions according to the degree of severity are summarized in Table 2. Mild, moderate and severe symptoms were seen in $35.2 \%, 41.1 \%$ and $23.5 \%$, respectively. Upper outer quadrant was the most common localization $(n=8,38 \%)$ followed by upper inner $(n=5,23.8 \%)$, lower inner $(n=4,19 \%)$ and lower outer $(n=4,19 \%)$ quadrants. Post-treatment severity scores are shown in Table 3. When compared with pre-treatment severity scores, there were very significant regression in all (clear, $76.4 \%$, mild, $17.6 \%$, moderate $3.9 \%$, severe $1.9 \%, \mathrm{p}<0.001$ for each). The overall success rate (total clearance plus mild signs) of treatment was $94.1 \%$. Images of patients before and after treatment are shown in Figures 1 and 2.

The survey results revealed that all patients applied SJW topically as instructed (100\%). There was no important side effect, except burning sensations $(n=2,9.5 \%)$. All patients were satisfied with the treatment $(100 \%)$.

\section{Discussion}

IGM is a recurrent benign disease usually affects women of child-bearing age, and other predisposing factors are lactation, inflammatory diseases, diabetes, smoking habit and antidepressant or oral contraceptive use. It usually presents with a tender, firm mass, and is often associated with inflammation of the skin. Secondary infection is common, and treatment is abscess drainage (if present), and empiric antibiotics. ${ }^{6,9}$ Although most of the masses respond well to the corticosteroids, and get smaller or disappear, skin lesions may still persist, especially in patients with persistent or recurrent disease. In the present series, the predisposing factors were not statistically significant $(\mathrm{p}>0.05$, each), except being in the reproductive age and having a history of lactation (90\% and 76\%, respectively). Hyperemia (100\%), scaling (61\%), induration (52\%) and ulcer $(28 \%)$ were the predominant skin lesions, since abscesses over $2-\mathrm{cm}$ and intractable skin fistulas were surgically managed with simple interventions at the outpatient clinic. Since we could not find any similar 
study, reporting the effects of topical SJW application on intractable skin lesions of IGM disease, we will not be able to compare our results.

Topical SJW applications have been used in some dermatologic disorders, such as egzema, psoriasis, burns etc., and promising results were reported. ${ }^{10,11}$ Furthermore, its use as a remedy for wounds, bruises and skin ulcers has been well-documented in the English-written literature. ${ }^{12-14}$ Of the constituents, flavonoids, hypericin and hyperforin have antimicrobial, antioxidant, anti-inflammatory and anticancer activities. ${ }^{1}$ Hyperforin also stimulates growth and differentiation of keratinocytes, and hypericin is a photosensitizer which can be used for selective treatment of skin cancer. ${ }^{3}$ Menichini et al. ${ }^{15}$ have suggested that SJW induces inhibition of free radicals and enhanced phototoxicity in human melanoma cells under ultraviolet (UV) light. Hypericin was able to suppress proliferation of human malignant melanoma cells, and the extract together with UV irradiation enhanced phototoxicity. This biological activity of antioxidant effects was combined with inhibition of nitric oxide production. It has also been suggested that other mechanism involves induction of apoptosis (programmed cell death) through the activation of caspases, which are cysteine proteases that trigger a cascade of proteolytic cleavage occurrences in mammalian cells. In an another study, Mansouri et al. ${ }^{16}$ showed that SJW ointment can help decrease the clinical flares of psoriasis. The authors suggested that its efficacy is probably related to its effect on lowering cytokines including tumor necrosis factor alpha (TNF $\alpha$ ). Yadollah-Damavand et al. ${ }^{17}$ have shown that topical hypericum perforatum improves tissue regeneration in full-thickness excisional wounds in diabetic rat model.

There are not many studies published on the properties of SJW, how it helps the wound healing process in detail. However, its anti-inflammatory, antioxidant and antimicrobial activities were already reported as well as fibroblast proliferation inducing effect. ${ }^{18-20} \mathrm{SJW}$ includes many more biologically active substances, although hypericin and hyperforin have the greatest medical activity. Other compounds, including the flavonoids, quercetin and kaempferol, also appear to have medical use. Ozturk et al. ${ }^{21}$ studied the wound healing activity of SJW on experimental embryonic fibroblasts. According to their findings, fibroblast density, collagen synthesis, and epithelial cell proliferation rate were increased in the presence of flavonoids and xanthones, which are among the constituents of SJW. In addition, flavonoids are known to reduce lipid peroxidation by preventing or slowing the onset of cell necrosis and improving vascularity. ${ }^{17}$ A novel study of Suntar et al demonstrated wound healing and antimicrobial activity of a topical formulation containing olive oil extract of SJW. ${ }^{22}$

In the present series, although high-dose oral corticosteroid treatment is very effective in reducing the mass, our previous experience with steroid pomads was disappointing. Since IGM usually presents with skin lesions, and some of them become persistent or intractable, we started to recommend topical SJW oil extracts from 2016. In the beginning, physical examination revealed hyperemia/erythema (100\%), scaling (61\%), induration (52\%) and fissure/ulcer (28\%). Distributions of the lesions according to the degree of severity were graded as mild, moderate and severe, and seen in $35 \%, 41 \%$ and $23 \%$ of the patients, respectively. When compared with pre-treatment severity scores, there were very significant regression (clear: $76.4 \%$, mild: $17.6 \%$, moderate: $3.9 \%$, severe: $1.9 \%$; $<<0.001$ for each). The overall success rate (total clearance plus mild signs) of treatment was $94 \%$, and all patients were satisfied with the treatment $(100 \%)$.

Previous studies including SJW use in depressive patients have shown that SJW is generally considered safe when used orally in appropriate doses. ${ }^{23}$ The most common adverse effects are gastrointestinal symptoms, allergic reactions, dizziness, restlessness, headache and dryness of the mouth, but these effects are generally mild and transient. Certain drug interactions with barbiturates, warfarin, statins, immunosuppressive drugs and chemotherapeutic agents have also been reported, depending on its effect on the liver P450 enzyme isotypes. ${ }^{23-25}$ However, there is not enough information about the safety of using SJW topically. In the present study, we just encountered mild burning sensations in two of the patients, but they tolerated well and continued using the drug to the end of sixth weeks.

In conclusion, SJW oil massage seems to be effective and promising in patients with persistent IGM-related skin lesions. However, further studies with higher volume of patients are needed to make a final decision. 


\section{Conflicts of interest}

There is no conflict of interest.

\section{References}

1. Wolfle U, Seelinger G, Schempp CM. Topical application of St. John's wort (Hypericum perforatum). Planta Med 2014; 80(2-3): 109-120.

2. Arsic I, Zugic A, Tadic V, Tasic-Kostov M, et al. Estimation of dermatological application of creams with St. John's Wort oil extracts. Molecules 2011; 17(1) :275-294.

3. Meinke MC, Schanzer S, Haag SF, Casetti F, et al. In vivo photoprotective and anti-inflammatory effect of hyperforin is associated with high antioxidant activity in vitro and ex vivo. Eur J Pharm Biopharm 2012; 81: $346-350$.

4. Arndt S, Haag SF, Kleemann A, Lademann J, et al. Radical protection in the visible and infrared by a hyperforin-rich cream - in vivo versus ex vivo methods. Exp Dermatol 2013; 22: 354-357.

5. Ozel L, Unal A, Unal E, Kara M, et al. Granulomatous mastitis: is it an autoimmune disease? Diagnostic and therapeutic dilemmas. Surg Today 2012; 42(8): 729-733.

6. Wang J, Zhang Y, Lu X, Xi C, et al. Idiopathic granulomatous mastitis with skin rupture: A retrospective cohort study of 200 patients who underwent surgical and nonsurgical treatment. J Invest Surg 2019; 1-6.

7. Thomas VM, Alexander SA, Bindal P, Vredenburgh J. Idiopathic granulomatous mastitis-A Mmystery yet to be unraveled: A case series and review of literature. Cureus 2020; 12(2): e6895.

8. Mehta BH, Amladi ST. Evaluation of topical $0.1 \%$ tazarotene cream in the treatment of palmoplantar psoriasis: an observer-blinded randomized controlled study. Indian J Dermatol 2011; 56(1): 40-43.

9. Li J. Diagnosis and treatment of 75 patients with idiopathic lobular granulomatous mastitis. J Invest Surg 2019; 32(5): 414-420.

10. Hoffmann J, Gendrisch F, Schempp CM, Wolfle U. New herbal biomedicines for the topical treatment of dermatological disorders. Biomedicines. 2020; 8(2): 27.

11. Seyhan N. Evaluation of the healing effects of hypericum perforatum and curcumin on burn wounds in rats. Evid Based Complement Alternat Med 2020; 2020: 6462956.

12. Whitehead AJ, Nelson NW, Brame LS, Champlin FR. Endemic North American plants as potentially suitable agents for wound cleaning under resource scarce conditions. Wilderness Environ Med 2019; 30(4): 401-406.

13. Egri O, Erdemir N. Production of Hypericum perforatum oil-loaded membranes for wound dressing material and in vitro tests. Artif Cells Nanomed Biotechnol 2019; 47(1): 1404-1415.

14. Barnes J, Anderson LA, Phillipson JD. St John's wort (Hypericum perforatum L.): A review of its chemistry, pharmacology and clinical properties. J Pharm Pharmacol 2001; 53(5): 583-600.

15. Menichini G, Alfano C, Marrelli M, Toniolo C, et al. Hypericum perforatum L. subsp. perforatum induces inhibition of free radicals and enhanced phototoxicity in human melanoma cells under ultraviolet light. Cell Prolif 2013; 46(2): 193-202.

16. Mansouri P, Mirafzal S, Najafizadeh P, Safaei-Naraghi Z, et al. The impact of topical Saint John's Wort (Hypericum perforatum) treatment on tissue tumor necrosis factor-alpha levels in plaque-type psoriasis: A pilot study. J Postgrad Med 2017; 63(4): 215-220.

17. Yadollah-Damavandi S, Chavoshi-Nejad M, Jangholi E, Nekouyian N, et al. Topical hypericum perforatum improves tissue regeneration in full-thickness excisional wounds in diabetic rat model. Evid Based Complement Alternat Med 2015; 2015: 245328. 
18. Wills RBH, Bone K, Morgan M. Herbal products: active constituents, modes of action and quality control. Nutrition Res Rev 2000; 13(1): 47-77.

19. Butterweck V. Mechanism of action of St John's wort in depression: what is known? CNS Drugs 2003; 17(8): 539-562.

20. Milosevic T, Solujic S, Sukdolak S. In vitro study of ethanolic extract of Hypericum perforatum L. on growth and sporulation of some bacteria and fungi. Turk J Biol 2007; 31(4): 237-241.

21. Ozturk N, Korkmaz S, Ozturk Y. Wound-healing activity of St. John's Wort (Hypericum perforatum L.) on chicken embryonic fibroblasts. J Ethnopharmacol 2007; 111: 33-39.

22. Suntar IP, Akkol EK, Ylmazer D, Baykal T, et al. Investigations on the in vivo wound healing potential of Hypericum perforatum L. J Ethnopharmacol 2010; 127: 468-477.

23. Klemow KM, Bartlow A, Crawford J, et al. Medical attributes of St. John's wort (Hypericum perforatum) In: Benzie IFF, Wachtel-Galor S, Editors. Herbal Medicine: Biomolecular and Clinical Aspects. 2nd edition. Boca Raton (FL): CRC Press/Taylor \& Francis; Chapter 11, 2011.

24. Henderson L, Yue QY, Bergquist C, Gerden B, et al. St John's wort (Hypericum perforatum): drug interactions and clinical outcomes. Br J Clin Pharmacol 2002; 54(4): 349-356.

25. Zhou S, Chan E, Pan SQ, Huang M, et al. Pharmacokinetic interactions of drugs with St John's wort. J Psychopharmacol 2004; 18(2): 262-276.

Table 1. Distribution of the lesions according to the degree of severity.

Severity Score Erythema Scaling Induration Fissure/ulcer

Clear 0 No sign No sign No sign No sign

Mild 1 Light to dark Fine, white Light palpable Superficial, few

pink elevation $(0.5 \mathrm{~mm})$

Moderate 2 Red (not dark) Diffuse, thick, Moderate Intermediate depth, most lesions definitive elevation multiple fissures

partly covered $(0.75 \mathrm{~mm})$

Severe 3 Deep red to Very thick coverage, Noticable height Numerous/

purple all lesions covered (at least $1 \mathrm{~mm}$ ) deep lesions

Table 2. Severity scores of lesions before treatment.

Severity Score Erythema Scaling Induration Fissure/ulcer Total

$(\mathrm{n}=21,100 \%)(\mathrm{n}=13,61.9 \%)(\mathrm{n}=11,52.3 \%)(\mathrm{n}=6,28.5 \%)(\mathrm{n}=51,100 \%)$

Clear 00000000

Mild $16(28.5 \%) 3(23 \%) 6(54.5 \%) 3(50 \%) 18(35.2 \%)$

Moderate $29(42.8 \%) 6(46.1 \%) 3(27.2 \%) 3(50 \%) 21(41.1 \%)$

Severe $36(28.5 .2 \%) 4(30.7 \%) 2(18.1 \%) 012(23.5 \%)$

Table 3. Severity scores of lesions after treatment.

Severity Score Erythema Scaling Induration Fissure/ulcer Total

$(n=21,100 \%)(n=13,61.9 \%)(n=11,52.3 \%)(n=6,28.5 \%)(n=51,100 \%)$ 
Clear $016(76.1 \%)^{* *} 11(84.6 \%)^{* *} 7(63.6 \%)^{* *} 5(83.3 \%)^{* *} 39(76.4 \%)^{* *}$

Mild $14(19 \%)^{*} 2(15.3 \%)^{*} 3(27 . .2 \%)^{* *} 0^{* *} 9(17.6 \%)^{* *}$

Moderate $21(4.7 \%)^{* *} 0^{* *} 0^{* *} 1(16.6 \%)^{* *} 2(3.9 \%)^{* *}$

Severe $30^{* *} 0^{* *} 1(9 \%)^{*} 01(1.9 \%)^{* *}$

$* \mathrm{P}<0.05$ : statistically significant, $\mathrm{p}<0.001$ : statistically highly significant

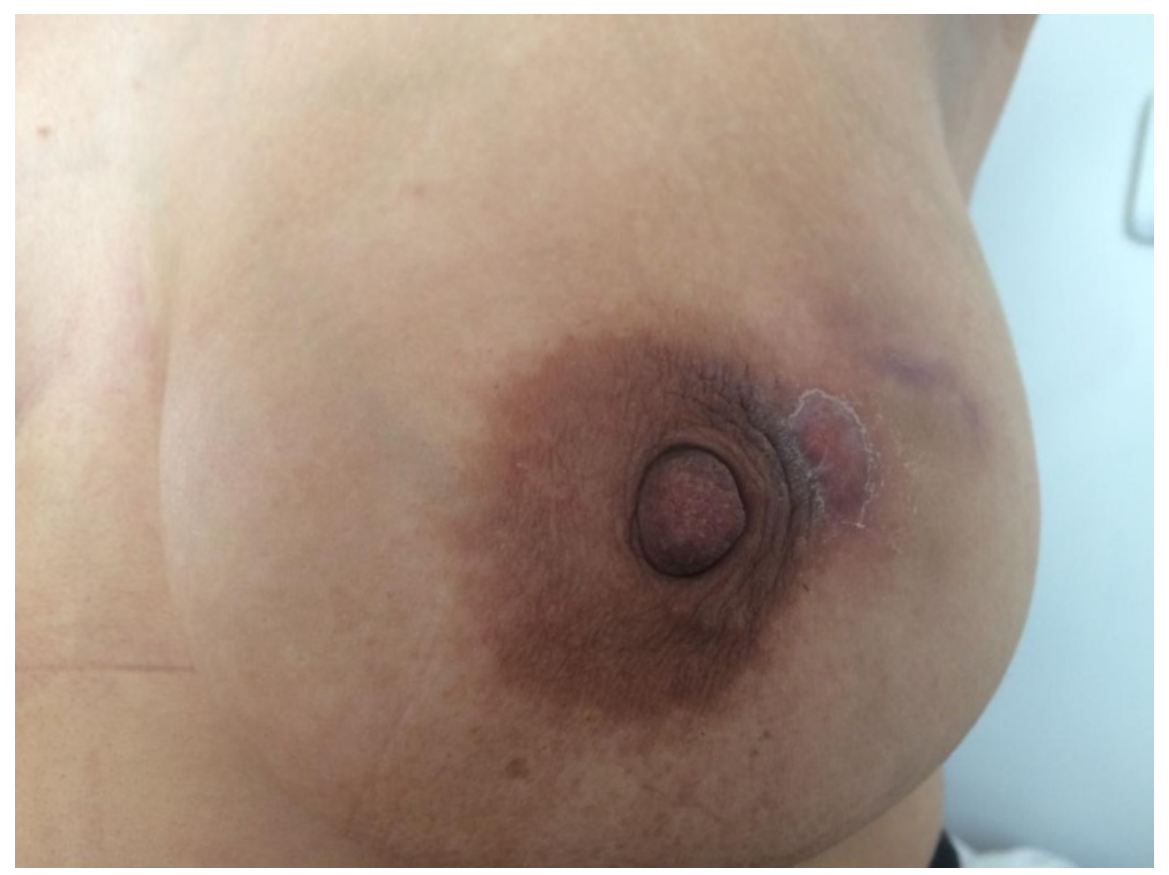

Figure 1. A persistent erythematous induration on the upper outer quadrant of left breast, even after completion of two cycles of high-dose oral corticosteroid together with broad spectrum antibiotic (Informed consent and permission taken from the patient M.U.) 


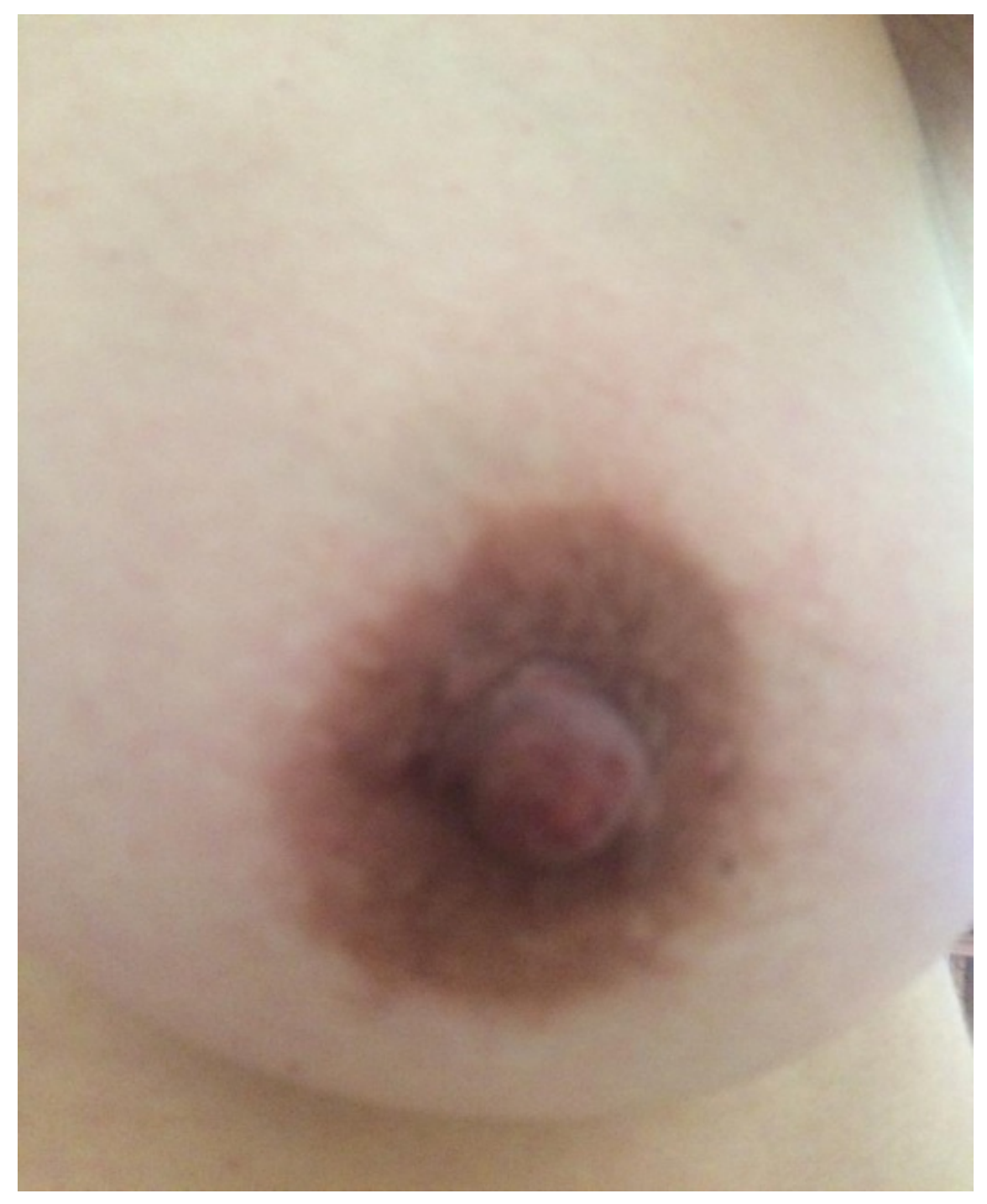

Figure 2. The lesion faded and disappeared after topical application of SJW oil macerate for 6 weeks (Informed consent and permission taken from the patient M.U.) 